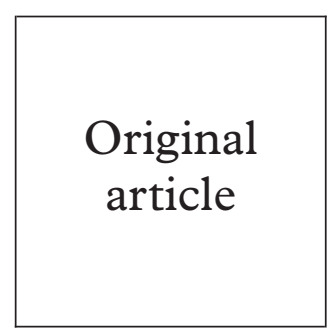

\title{
Passive sentinel surveillance system for sexually transmitted diseases in primary healthcare sites in Ethiopia, 1991-3
}

\author{
A Matteelli, A Kassa, A Gerbase, C Farina, M Ghidinelli, G Chatel, A Beltrame, W Feleke, \\ G Carosi
}

Background: In 1989 the ministry of health of Ethiopia launched an STD control programme to strengthen the STD case management capabilities at public health centres and hospitals. The programme included the introduction of a syndrome based system for notification of STD cases. We here report the data originated by the syndromic case reporting system under programme conditions.

Methods: $35(17 \%)$ of the total 225 hospitals and public health centres of Ethiopia were included in the programme. Information relevant to the years 1991 to 1993 was analysed at mid 1994.

Results: 32 clinical sites ( $91 \%$ of the total) provided at least one monthly report. The proportion of monthly reports received was $65 \%$ of those due, ranging from $51 \%$ in 1991 to $73 \%$ in 1992 and $42 \%$ in 1993. A total of 77294 consultations for STD related symptoms were recorded, including 70200 new cases, 6588 repeated consultations, and 506 partners of STD patients. Among first attendant patients $38459(52.7 \%)$ were males with a male to female ratio of $1: 1$. Urethral discharge and vaginal discharge were the leading cause of consultation among males $(58 \%)$ and females $(64 \%)$ respectively. The frequency of genital ulcer diseases was $26 \%$ among males and $15 \%$ among females. Inguinal adenopathy in the absence of genital ulcers was also frequent, accounting for $10 \%$ of consulting males and $5 \%$ of females. Based on Gram stain, gonorrhoea was identified in $64 \%$ of the cases of urethral discharge, while trichomoniasis and candidiasis were identified by wet mount in $28 \%$ and $16 \%$ of the cases of vaginal discharge respectively.

Conclusions: STDs are a common cause of consultation at public health centre sites in Ethiopia. A syndromic case reporting system proved to be efficient and produced valuable information to initiate assessment of the problem and to set up bases for monitoring trends of STD morbidity. (Sex Transm Inf 2000;76:131-133)

Keywords: sexually transmitted diseases; case notification; Ethiopia

Clinic of Infectious and Tropical Diseases, University of Brescia, Brescia, Italy

A Matteelli

$M$ Ghidinelli

G Chatel

A Beltrame

G Carosi

\section{STD Division,}

Ministry of Health,

Republic of Ethiopia

A Kassa

M Ghidinelli

W Feleke

World Health

Organisation Office of

HIVIAIDS and STD

A Gerbase

Microbiology Institute, A O 'Ospedali Riuniti

di Bergamo',

Bergamo, Italy

C Farina

Correspondence to: Alberto Matteelli, Clinic of Infectious and Tropical

Diseases, University of

Brescia, Brescia, Italy

forleo@master.cci.unibs.it

Accepted for publication

21 December 1999

\section{Introduction}

Sexually transmitted diseases (STDs) are a major global cause of acute illness, infertility, long term disability, and death. ${ }^{1}$ In subSaharan Africa the estimates of STD rates are significantly higher compared with industrialised nations, mainly as a consequence of limited economic resources and poor access to medical services. ${ }^{2}$ The importance of STDs as a public health problem has been amplified by the understanding of the role these diseases have in driving the HIV epidemic in tropical Africa $^{34}$ and by the demonstration that effective STD control may significantly reduce the incidence of HIV. ${ }^{5}$

In Ethiopia the epidemiology division of the ministry of health runs a surveillance system based on passive reporting. Considering lack of laboratory resources most of the diagnoses are made only on a clinical basis. STDs ranked sixth, constituting $4.7 \%$ of all reportable communicable diseases in 1986-7.6 Gonorrhoea was estimated to be the most widespread STD with an incidence of 132/1000 population, while syphilis and chancroid incidences were estimated as 13.4 and 13.8 respectively. ${ }^{7}$ Since the health system covers only partially the real STD population, probably those rates have heavily underestimated the problem. Though charged by important limitations, an STD syndromic case reporting system may provide valuable epidemiological indicators of STD incidence and incidence trends. Cases of urethral discharge and genital ulcers are particularly useful because they usually reflect recently acquired infections that are specifically transmitted via the sexual route. ${ }^{8}$ Syndromic reports of such cases may be used as indicators of the impact of control interventions.

A national programme for STD prevention and control was launched in Ethiopia in July 1989. A specific information system based on reports of STD syndromes was developed and introduced in the intervention area at the beginning of 1991. This paper presents the data originated under programme conditions by the STD information system during the period 1991 to 1993 .

\section{Intervention area and methods}

AREA OF THE INTERVENTION

From 1989 to 1994 the syndromic case management strategy was introduced in 35 public healthcare sites, which represent approximately $17 \%$ of the 225 hospitals and health centres of Ethiopia. Both health centres 


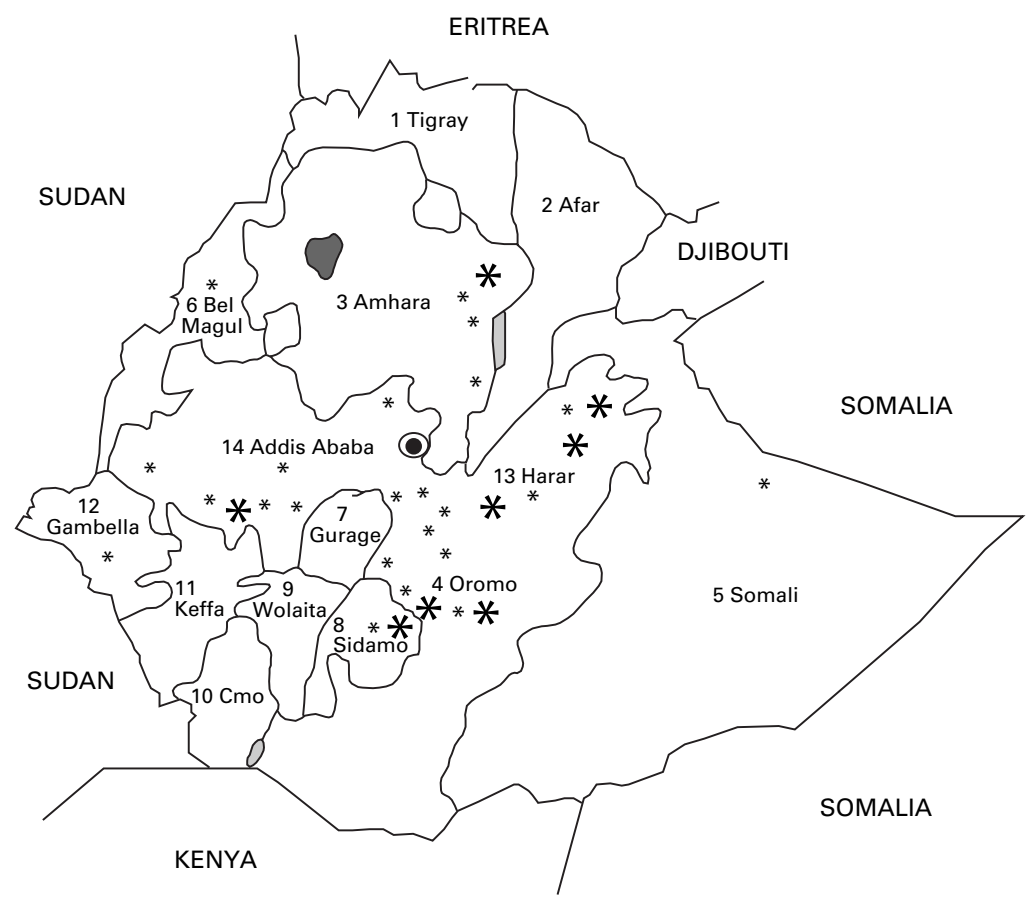

* Health centres

* Outpatient department of district hospital

Figure 1 Location of 35 public healthcare sites in Ethiopia (four centres in Addis Ababa).

and outpatient departments of district hospitals were included; selection of the sites was based on convenience and all sites were receiving typical public health centre patients. Given the conditions of the Ethiopian health system at that time it was not possible to determine the catchment area of those clinics. Figure 1 shows the map of the country (administrative regions are those of 1994) with the location of the 35 clinical sites. Half of the sites started the activities in 1991, while the remainder joined the project in 1992 .

STD CASE MANAGEMENT AND REPORTING SYSTEM Following the national policies for health development, STD services were integrated into primary healthcare services. STD case management was based on a syndromic approach. Guidelines were developed, STD drugs were regularly distributed, monitoring and supervision were ensured by the STD division. An information system was set up to collect data on numbers of STD syndromes diagnosed. The analysis of STD notifications was conducted on the reports obtained by August 1994, while laboratory results were limited to the period January 1991 to June 1992.

\section{DEFINITION OF SYNDROMES}

Syndromes were defined as follows: urethral discharge in males as the presence of exudate in the anterior urethra; vaginal discharge in females as the presence of exudate from the vagina which was abnormal in colour, odour, and/or amount; genital ulcers as a loss of continuity of genital skin and/or mucosae; buboes as the enlargement of the lymph glands in the groin area; warts as raised growths on the genitalia and the perianal area. Lower abdominal pain in non-pregnant women was reported after exclusion of common bowel, urinary, and surgical causes.

STD LABORATORY TESTS FOR PATIENTS WITH URETHRAL AND VAGINAL DISCHARGE

Case management algorithms for urethral discharge in males included a Gram stain for detection of intracellular diplococci (ICDC) and leucocytes (WBC). A wet mount preparation for detection of Trichomonas vaginalis and yeasts was advised for cases of vaginal discharge. In all selected clinical sites basic STD laboratory facilities were maintained by provision of equipment, training of personnel, and regular supply of reagents.

\section{Results}

EFFECTIVENESS OF CASE REPORTING SYSTEM

The number of clinical sites that provided at least one clinical report during the period 1991-3 was 32 (91\% of included sites). The overall proportion of reports produced was $65 \%$, ranging from $51 \%$ in 1991 to $73 \%$ in 1992 and $42 \%$ in 1993 . The number of centres that produced reports decreased from 32 in 1992 to 25 in 1993.

\section{SYNDROMIC STD CASE REPORTS}

Overall, 77294 consultations for STD related problems were recorded during the 3 year observation period, including 70200 new patients, 6588 repeated consultations, while the number of partners passively referred by the index case and treated was 506. The number of notifications grew from 14090 in 1991 to 30128 in 1992 , but later decreased to 25982 in 1993. The average number of notified patients per month per centre decreased from 148 in 1991 to 111 in 1992, and eventually increased to 159 in 1993. Among first attendants $38459(52.7 \%)$ were males with a male to female ratio of $1: 1$. Urethral discharge was the leading cause of consultation among males (58\%), while vaginal discharge was reported by the majority of consulting females $(64 \%)$. The frequency of genital ulcer diseases (GUD) was $26 \%$ among males and $15 \%$ among females. Inguinal adenopathy in absence of genital ulcers was also frequent, accounting for $10 \%$ of consulting males and $5 \%$ of females. Lower abdominal pain was the cause of consultation in $8 \%$ of the women.

\section{STD LABORATORY TESTS FOR PATIENTS WITH}

URETHRAL AND VAGINAL DISCHARGE

Results from 3766 Gram stain preparations on urethral samples and 3435 wet mount preparations on vaginal samples were available: $64 \%$ of the urethral samples were positive for ICDC, $9 \%$ had WBC but not diplococci, and $27 \%$ were negative for either finding. Vaginal samples were positive for Trichomonas vaginalis in $28 \%$ of the cases and yeasts in $16 \%$.

\section{Discussion}

This report provides the first community generated data on the frequency distribution of 
STD syndromes in Ethiopia. A syndromic case reporting system proved to be efficient and produced valuable information to initiate assessment of the problem and to set up a basis for monitoring trends of STD morbidity despite ongoing civil unrest, political changes, and administrative reorganisation. The fact that STDs are a common health problem in Ethiopia is suggested by several observations. Firstly, urethral discharge, a sign which is quite specific for sexually transmitted pathogens, was present in over a quarter of attendants with genital symptoms. Gonorrhoea is the largely predominant cause of urethral discharge in this setting, as shown by the $65 \%$ positivity rate for ICDC at Gram stain. These data are consistent with previous observations on the aetiology of urethral discharge among STD patients in Addis Ababa. ${ }^{9}$ Secondly, the frequency of GUD was extremely high representing $15 \%$ of females and $26 \%$ of the males consulting for genital symptoms. GUD are associated with the highest relative risk for increased HIV transmission. ${ }^{3}$ It would be important to assess the relative aetiologies of genital ulcers in Ethiopia: in Uganda most genital ulcers were found not to be caused by either Treponema pallidum or Haemophilus ducreyi, ${ }^{10}$ which are the two agents currently managed for in the case management algorithms. Thirdly, the proportion of Trichomonas vaginalis infections was higher than that of yeasts among women presenting with vaginal discharge $(28 \%$ of positive wet mount tests compared with $16 \%$ ), suggesting that a significant proportion of those women indeed had a true STD.

The AIDS epidemic involved Ethiopia in the second half of the 1980s; by the end of June 1994 the cumulative number of notified AIDS cases in the country was 13644 and rose to 21569 by the end of 1997. ${ }^{11}$ The HIV seroprevalence among blood donors had increased from $3.6 \%$ in 1987 to $6.2 \%$ in 1991 , and the HIV prevalence rates among commercial sex workers in Addis Ababa had increased from $35.9 \%$ in 1988 to $69.4 \%$ in 1991 (Minis- try of Health, unpublished data). The evidence of a high prevalence of STD in the general population supports the crucial part that these conditions might have played in fuelling the HIV epidemic and strengthens the need for effective control interventions.

This project received financial support from the European Commission, under the 5th European Development Fund, contract no 5710-9495-005.

Contributors: A Matteelli analysed and interpreted data and wrote the manuscript; A Kassa supervised collection of clinical data and commented on draft of the paper; A Gerbase contributed to data interpretation and preparation of the manuscript; C Garina supervised collection of laboratory data; $M$ Ghidinelli Farina supervised collection of laboratory data; $M$ Ghidinelli
designed the information system as STD consultant; G Chatel designed the information system as STD consultant; G Chatel was involved in data processing and analysis; A Beltrame was
involved in data processing and analysis; W Feleke designed the information system as director of STD division; G Carosi was adviser to the design of information system and overall study supervisor.

1 Gerbase AC, Rowley JT, Mertens TE. Global epidemiology of sexually transmitted diseases. Lancet 1998;351 (supp III): $2-4$

2 Meheus A, Shultz K, Cates W. Development of prevention and control programs for sexually transmitted diseases in developing countries. In: K Holmes, P March, eds. Sexually transmitted diseases. 2nd ed. New York: McGraw-Hill, 1990: 1041.

3 Cohen MS. Sexually transmitted diseases enhance HIV transmission: no longer a hypothesis. Lancet 1998;351 (suppl III): 5-7.

4 Hitchcock P, Fransen L. Preventing HIV infection: lessons from Mwanza and Rakai. Lancet 1999;353:513-5.

5 Grosskurth H, Mosha F, Todd J, et al. Impact of improved treatment of sexually transmitted diseases on HIV infection in rural Tanzania. Randomised control trial. Lancet 1995;346:530-6.

6 Ministry of Health of Ethiopia. Comprehensive health service directory, 1986 and 1987 planning and programming bureau. Addis Ababa: Ministry of Health, 1988:4-18.

7 Asefa G. Magnitude of STDs problems in Ethiopia. In: Proceedings 1st National Seminar for the launching of the STD National Control Programme. Addis Ababa of the STD National Control Programme. Addis Ababa, Ethiopia, 25-26 January 1990. Addis Ababa, Ethiopia: Ministry

United Nations Programme on HIV/AIDS and World Health Organisation. Guidelines for sexually transmitted diseases surveillance. UNAIDS/WHO document STD GLN/02. Geneva: WHO, 1998.

9 Desta S, Feleke W, Mohamed Y, T et al. Prevalence and therapeutic response of male acute uncomplicated urethritis: relevance to STD and AIDS control in Ethiopia. VI International Conference of AIDS in Africa, Dakar, 1991: abstract MO 129.

10 Wawer, MJ, Sewankambo NK, Serwadda D, et al. Control of sexually transmitted diseases for AIDS prevention in Uganda: a randomised community trial. Lancet 1999;353. 525-35.

11 World Health Organisation. Global AIDS surveillance. Wkly Epidem Rec 1998;73:193. 